\title{
Preparation of distilled and purified continuous-variable entangled states
}

\author{
BORIS HAGE ${ }^{1}$, AIKO SAMBLOWSKI ${ }^{1}$, JAMES DIGUGLIELMO ${ }^{1}$, ALEXANDER FRANZEN ${ }^{1}$, \\ JAROMÍR FIURÁŠEK ${ }^{2}$ AND ROMAN SCHNABEL ${ }^{1 *}$
}

\author{
${ }^{1}$ Albert-Einstein-Institut, Max-Planck-Institut für Gravitationsphysik and Leibniz Universität Hannover, Callinstr. 38, 30167 Hannover, Germany \\ ${ }^{2}$ Department of Optics, Palacký University, 17. listopadu 50, 77200 Olomouc, Czech Republic \\ *e-mail: roman.schnabel@aei.mpg.de
}

Published online: 2 November 2008; doi:10.1038/nphys1110

The distribution of entangled states of light over long distances is a major challenge in the field of quantum information. Optical losses, phase diffusion and mixing with thermal states lead to decoherence and destroy the non-classical states after some finite transmission-line length. Quantum repeater protocols ${ }^{1,2}$, which combine quantum memory ${ }^{3}$, entanglement distillation ${ }^{4,5}$ and entanglement swapping ${ }^{6}$, were proposed to overcome this problem. Here we report on the experimental demonstration of entanglement distillation in the continuous-variable regime ${ }^{7-9}$. Entangled states were first disturbed by random phase fluctuations and then distilled and purified using interference on beam splitters and homodyne detection. Measurements of covariance matrices clearly indicate a regained strength of entanglement and purity of the distilled states. In contrast to previous demonstrations of entanglement distillation in the complementary discrete-variable regime ${ }^{10,11}$, our scheme achieved the actual preparation of the distilled states, which might therefore be used to improve the quality of downstream applications such as quantum teleportation ${ }^{12}$.

Quantum information makes use of the special properties of quantum states to improve the quality of communication and information processing tasks. Generally, a quantized field can be described by the number operator or alternatively by two non-commuting position and momentum-like operators. The corresponding measurement results have either discrete or continuous spectra and form the basis of discrete-variable or continuous-variable quantum information, respectively. In the regime of continuous variables, entangled states of light can be deterministically generated in optical parametric amplifiers (OPAs), precisely manipulated with linear optics and measured with very high efficiency in balanced homodyne detectors. These entangled two-mode squeezed states show Gaussian probability distributions and were used for quantum teleportation ${ }^{12}$ and entanglement swapping ${ }^{13,14}$. Entangled states of the collective spins of two atomic ensembles analogous to two-mode squeezed states have been generated ${ }^{15}$, storage of quantum states of light in an atomic memory has been demonstrated ${ }^{3}$ and teleportation from light onto an atomic ensemble has been reported ${ }^{16}$. High-speed quantum cryptography with coherent light beams and homodyne detection has been demonstrated ${ }^{17}$. All these spectacular achievements reveal the great potential of this approach to quantum information processing.
A missing piece in this toolbox has been a feasible protocol for entanglement distillation and purification. Entanglement distillation $^{4,5}$ extracts from several shared copies of weakly entangled mixed states a single copy of a highly entangled state using only local quantum operations and classical communication between the two parties sharing the states. This turned out to be a very challenging task for continuous-variable states, because it was proved that it is impossible to distil Gaussian entangled states by means of the experimentally accessible Gaussian operations $^{18,19}$. However, a whole class of important decoherence processes give rise to non-Gaussian noise and therefore produce non-Gaussian entangled states. It has been shown ${ }^{9}$ that in this case the entanglement distillation can be carried out using only interference on beam splitters, balanced homodyne detection and conditioning on the measurement outcomes. This was recently experimentally confirmed by successful demonstrations of distillation and purification protocols for single squeezed modes that suffered from de-Gaussifying noise $\mathrm{e}^{20-22}$.

This work experimentally demonstrates the first distillation protocol for continuous-variable entangled states. Our protocol uses two copies of phase-diffused ${ }^{22,23}$, therefore mixed, entangled states showing a positive, but non-Gaussian, Wigner function. Our protocol enhances the entanglement and purity of the decohered states and represents a single step of an iterative Gaussification scheme $^{7,8}$ that asymptotically converts any input state into a Gaussian one. Moreover, if combined with a single de-Gaussifying operation such as the recently demonstrated single-photon subtraction from squeezed beams $s^{24-26}$, it would provide a generic continuous-variable entanglement purification and distillation scheme $e^{7,8}$, that is capable, for instance, of suppressing the detrimental effect of losses in quantum state transmission.

A schematic sketch of our protocol is shown in Fig. 1. Two OPAs provided two continuous-wave light fields that carried squeezed states of light. Both squeezed states were mixed with vacuum states on beam splitters with $50 \%$ power reflectivity to prepare two copies of so-called v-class ${ }^{27}$ entangled states. Entanglement prepared in this way is not the strongest possible but is effectual for this proof-of-principle experiment. All four resulting beams of the two copies of entangled states were transmitted to two parties, Alice (A) and Bob (B), through four channels showing independent phase noise. The noisy channels were realized by quasirandom electromechanical actuation of mirror positions in 


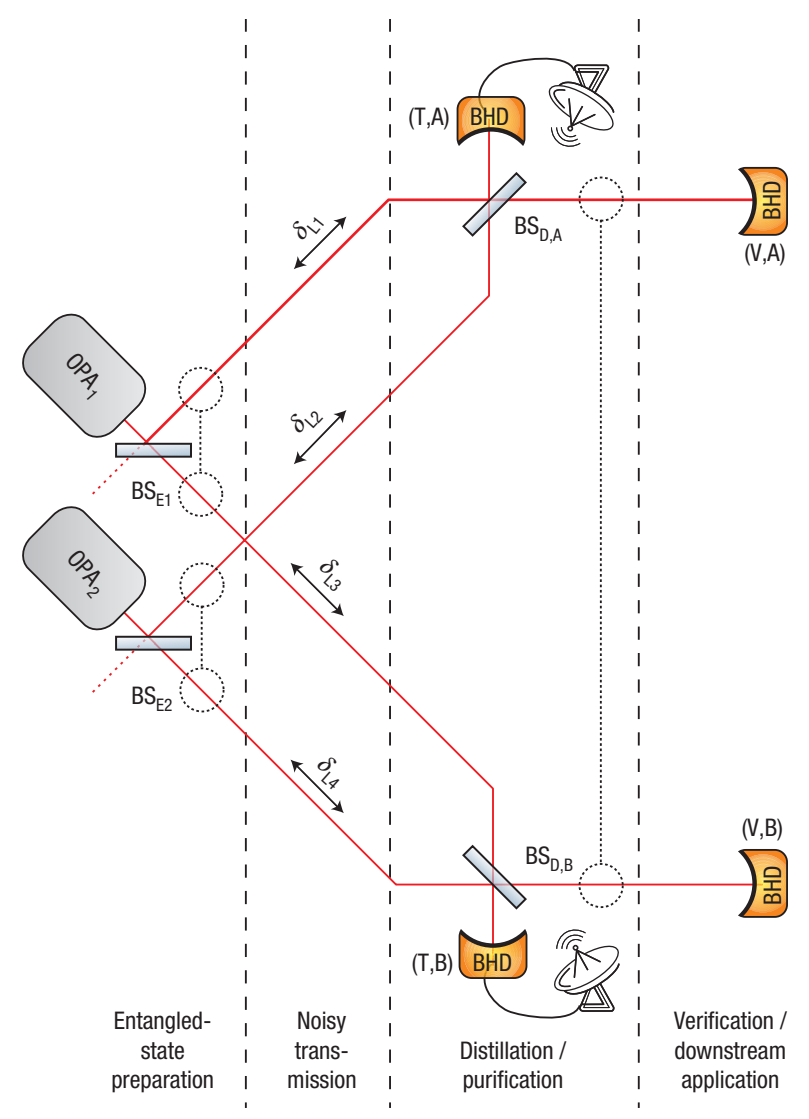

Figure 1 Experimental set-up. OPA, optical parametric amplifier; $\mathrm{BS}_{\mathrm{D}}$, distillation beam splitter; $\mathrm{BS}_{\mathrm{E}}$, entangling beam splitter; $\mathrm{BHD}$, balanced homodyne detector. $\delta_{L i}$ indicate independently fluctuating optical path lengths. The combination of both $B H D_{T, A / B}$ provided the trigger signal for successful distillation, whereas $B H D_{V, A / B}$ were used for its independent verification. The latter were not necessary for the distillation protocol to work.

the beam paths ${ }^{22,23}$ to mimic the phase noise introduced, for example in optical fibres. The phase fluctuations applied showed a Gaussian distribution, hence the standard deviation of the noise $\sigma_{\text {pn }}$ provided a complete characterization of its strength.

Alice and Bob each received two beams which they overlapped on a balanced beam splitter $\left(\mathrm{BS}_{\mathrm{D}, \mathrm{A}}\right.$ and $\mathrm{BS}_{\mathrm{D}, \mathrm{B}}$ in Fig. 1). The mean phase was controlled such that the initial quadrature phases lined up. The two output ports of each beam splitter were connected to a total of four balanced homodyne detectors (BHDs). Each of the BHDs could be set to observe an arbitrary quadrature, in particular the amplitude quadrature $X$ or the conjugate phase quadrature $P$. The detectors $\mathrm{BHD}_{\mathrm{T}, \mathrm{A}}$ and $\mathrm{BHD}_{\mathrm{T}, \mathrm{B}}$ were used to generate trigger events discriminating the success or failure of preparation of a distilled state, whereas $\mathrm{BHD}_{\mathrm{V}, \mathrm{A}}$ and $\mathrm{BHD}_{\mathrm{V}, \mathrm{B}}$ were used to independently verify the performance of the protocol. The latter two were therefore not part of the actual protocol and could be replaced by an arbitrary experiment. The fringe visibilities on all four BHDs as well as on $\mathrm{BS}_{\mathrm{D}, \mathrm{A} / \mathrm{B}}$ were between $97.1 \%$ and $98.2 \%$. In our experiment both trigger detectors $\mathrm{BHD}_{\mathrm{T}, \mathrm{A} / \mathrm{B}}$ were set to measure the initially squeezed amplitude quadratures $X_{\mathrm{T}, \mathrm{A}}$ and $X_{\mathrm{T}, \mathrm{B}}$, respectively. We chose the trigger condition determining the success of the distillation protocol to be ${ }^{9}$

$$
\left|X_{\mathrm{T}, \mathrm{A}}+X_{\mathrm{T}, \mathrm{B}}\right|<Q
$$
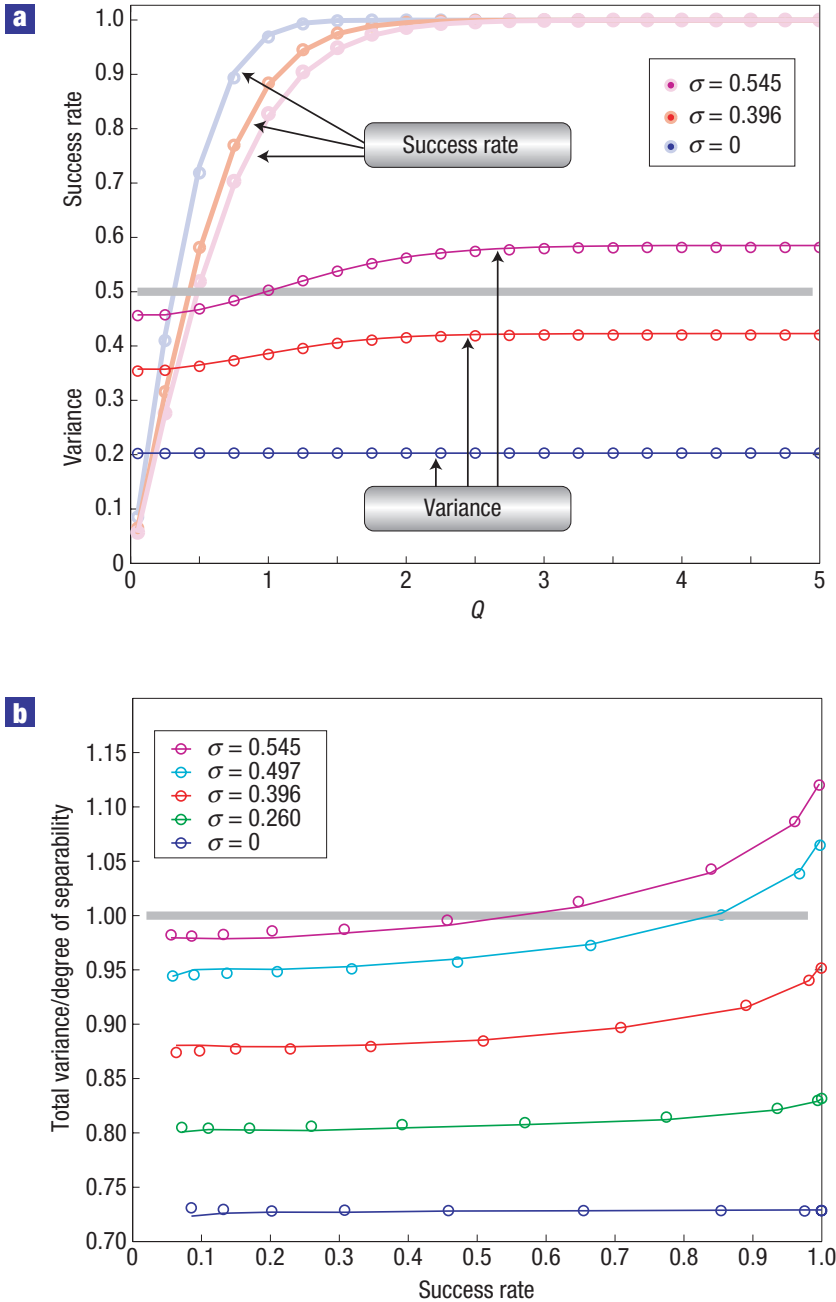

Figure 2 Non-local quadrature variance and total variance of the distilled states. a, Variance of $\left(X_{V, A}+X_{V, B}\right)$ and corresponding success rates versus threshold value $Q$ for different strengths of the phase fluctuations $\sigma_{\mathrm{pn}}$. Circles, measurement; line, simulation. b. The total variance / plotted against the success rate can be seen as a measure for the performance of our protocol. The grey lines indicate the vacuum noise level or separability boundary, respectively. For a success rate as high as 0.5 the protocol deploys nearly its full potential.

where $Q$ was a certain threshold whose value could be tuned in order to vary the selectivity of the protocol.

Figure $2 \mathrm{a}$ shows the variance of the non-local EinsteinPodolsky-Rosen (EPR)-like quadrature operator $\left(X_{\mathrm{V}, \mathrm{A}}+X_{\mathrm{V}, \mathrm{B}}\right)$ of the successfully distilled state and its corresponding probability of successful preparation versus threshold value $Q$ for three different strengths of the phase fluctuations $\sigma_{\mathrm{pn}}$. The lower the threshold was set, the more selectively our protocol worked. The variance of the orthogonal EPR-like quadrature operator $\left(P_{\mathrm{V}, \mathrm{A}}-P_{\mathrm{V}, \mathrm{B}}\right)$ was also reduced, which is not shown explicitly but becomes evident from our results shown in Fig. 2b. In this case the effect was rather small and limited by the vacuum noise value of $1 / 2$, because v-class ${ }^{27}$ entangled states were used in our experiment.

Figure $2 \mathrm{~b}$ shows the total variance $I$ of the distilled state versus its preparation success rate for five different strengths of phase noise. The total variance quantifies the quantum correlations between two modes and the degree of inseparability of the joint 


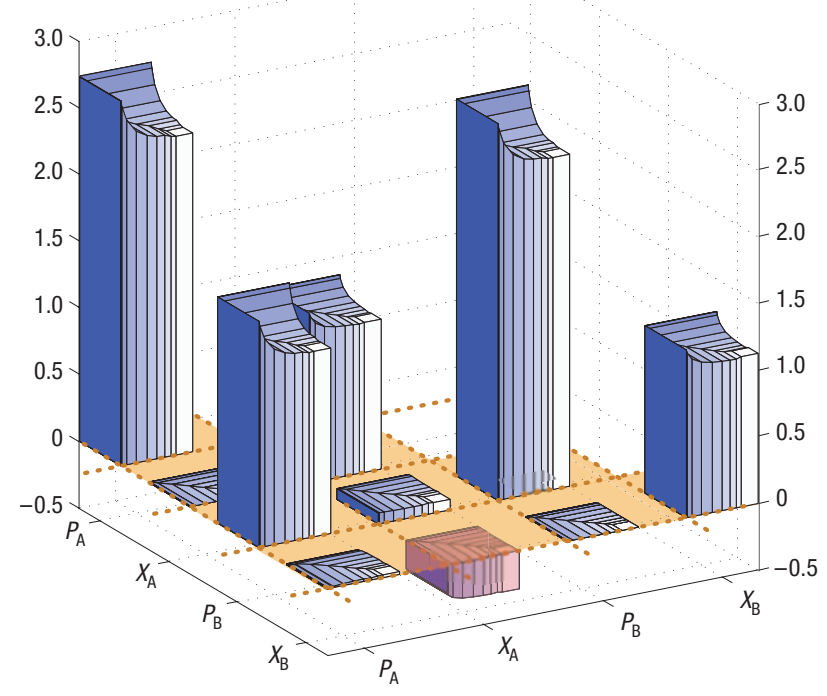

Figure 3 Reconstructed covariance matrices of distilled entangled states. The lower left triangle of this matrix (highlighted in brown) contains its ten significant elements. The sub-bars represent the results of our distillation protocol for different threshold values $Q$ in the case of $\sigma_{\mathrm{pn}}=0.497$. The base area of each sub-bar represents the corresponding success rate. The vacuum reference for this plot is given by the unit matrix.

state (see Methods section). If $I<1$ then the state is not separable and is therefore entangled ${ }^{28}$. We can see that the distillation and purification protocol reduces the total variance $I$ of the phase-diffused state. For the two strongest levels of phase noise, the total variance shows no non-classical behaviour without the distillation protocol, but was reduced below the unity boundary after applying the distillation protocol. Lower values of $Q$ result in a stronger distillation effect but also in a reduced success probability. It is promising to find that for a success rate as high as 0.5 the protocol had nearly developed its full potential. Note that the total variance of the entangled state before applying phase noise was evaluated to be $I=0.725$.

As the homodyne detectors can measure arbitrary quadrature phases, we carried out a tomographic reconstruction of the covariance matrices of the distilled two-mode entangled states. We followed the reconstruction procedure that is described in detail in our previous work ${ }^{27}$ and is based on joint measurements of quadrature operators of modes $\mathrm{V}, \mathrm{A}$ and $\mathrm{V}, \mathrm{B}$ for four different settings of the BHDs. From these data we determined the most significant eight of the ten independent parameters of the covariance matrix, namely the variances of four quadratures $X_{\mathrm{V}, \mathrm{A}}$, $P_{\mathrm{V}, \mathrm{A}}, X_{\mathrm{V}, \mathrm{B}}, P_{\mathrm{V}, \mathrm{B}}$ and covariances between all pairs of quadratures of Alice and Bob. The intramodal correlations were ignored, because the deviation from zero was too small to be measured within the given accuracy of the quadrature phase. The resulting covariance matrices are plotted in Fig. 3 for ten different success rates. With decreasing success rate the distillation becomes stronger, as shown by the reduction of the quadrature variances, that is, diagonal elements of the covariance matrix. Moreover, the anticorrelation between $X_{\mathrm{V}, \mathrm{A}}$ and $X_{\mathrm{V}, \mathrm{B}}$ was slightly enhanced. Consequently, the squeezing of the non-local quadrature $X_{\mathrm{V}, \mathrm{A}}+X_{\mathrm{V}, \mathrm{B}}$ was enhanced by the distillation.

The determinant $D=\operatorname{det}(\gamma)$ is an important characteristic of a covariance matrix $\gamma$. The purity $\Pi$ of a Gaussian state $\rho$

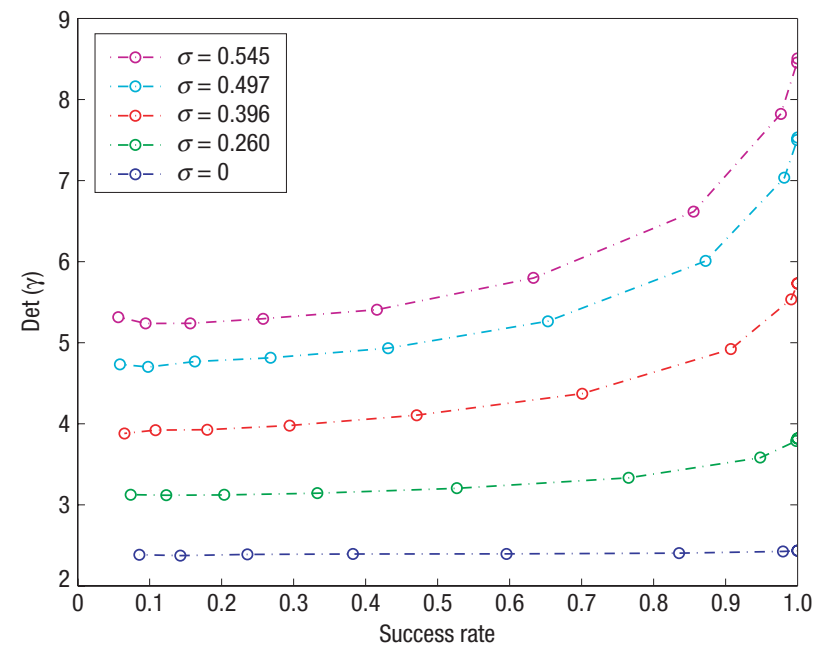

Figure 4 Determinants of covariance matrices of distilled states. The determinant $D$ versus the success rate of the distillation protocols for five different levels of phase noise. The decrease of the determinant $D$ towards the left is an indication of increased purity and Gaussification of the distilled state.

defined as $\Pi=\operatorname{Tr}\left[\rho^{2}\right]$ is given by $\Pi=1 / \sqrt{D}$. In Fig. 4 we plot the dependence of $D$ on the success rate of the distillation protocol. The distillation reduces $D$, which is a very strong indication of the increased purity of the (still slightly non-Gaussian) distilled state, because a generalized Heisenberg uncertainty relation implies that $D \geq 1$ and $D=1$ holds only for pure Gaussian states. In our experiment the determinant was generally limited from below to $D \gtrsim 2$ owing to optical losses, for example due to non-perfect quantum efficiencies of the homodyne detectors. The anticipation that the distilled states should show not only purification but also Gaussification was indeed confirmed by fitting Gaussian functions to the measurement data before and after the distillation protocol.

The protocol demonstrated here is able to distil entangled states from a decohered ensemble that suffered from phase noise in the transmission channel, for example due to thermally excited refractive-index fluctuations or Brillouin scattering. Because of its quantum nature, our protocol can counteract phase diffusion in cases where any classical channel probing would fail, for example if the phase fluctuations are intensity dependent and arise from quantum fluctuations in the photon number of the transmitted states. Our protocol provides two open ports that output the distilled entangled states, and is therefore unconditionally useful for arbitrary downstream quantum information applications that involve the second order moments of quadrature operators. An example is the Braunstein-Kimble teleportation protocol ${ }^{12}$.

The reported entanglement distillation, purification and Gaussification protocol can be iterated ${ }^{7,8}$ and combined with already experimentally demonstrated single-photon subtraction ${ }^{24-26}$, quantum memory ${ }^{3}$ and entanglement swapping $^{13,14}$ to build a continuous-variable quantum repeater. Our experiment is thus an important enabling step towards truly long-distance quantum communication with continuous variables.

\section{METHODS}

\section{GENERATION AND DETECTION OF SQUEEZED STATES}

Both OPAs were constructed from second-order nonlinear crystals $\left(\mathrm{MgO}: \mathrm{LiNbO}_{3}\right)$ inside a degenerate doubly resonant cavity ${ }^{23}$ and were pumped with frequency-doubled laser beams from a monolithic solid-state 
laser (Nd:YAG) operated at $1,064 \mathrm{~nm}$. The OPAs emitted continuous-wave squeezed beams with observed values of about $4.5 \mathrm{~dB}$ of squeezing and $8 \mathrm{~dB}$ of antisqueezing at Fourier frequencies around $7 \mathrm{MHz}$.

All four output beams of the distillation protocol were measured by BHDs, each consisting of a balanced beam splitter, a coherent local oscillator beam at 1,064 $\mathrm{nm}$ and two photodiodes. The photocurrents produced by the photodiodes were subtracted and amplified by home-made electronics. The signals from the four BHDs were electronically mixed with a $7 \mathrm{MHz}$ local oscillator. The intermediate signals were then anti-alias filtered at $400 \mathrm{kHz}$, synchronously sampled with $1 \mathrm{MHz}$ and postprocessed to accomplish and verify the distillation protocol.

\section{ENTANGLEMENT DETECTION AND QUANTIFICATION}

The entanglement of the distilled two-mode squeezed output state can be conveniently verified by the Duan criterion ${ }^{28}$. This criterion involves the sum of variances of two non-local, EPR-like quadrature operators, the so-called total variance, $I=\left\langle\left(\Delta X_{+, \mathrm{V}}\right)^{2}\right\rangle+\left\langle\left(\Delta P_{-, \mathrm{V}}\right)^{2}\right\rangle$, where $X_{+, \mathrm{V}}=X_{\mathrm{V}, \mathrm{A}}+X_{\mathrm{V}, \mathrm{B}}$ and $P_{-, \mathrm{V}}=P_{\mathrm{V}, \mathrm{A}}-P_{\mathrm{V}, \mathrm{B}}$. The state is entangled if $I<1$, where the vacuum state, with variance of arbitrary single-mode quadrature $1 / 4$, corresponds to a value of $I=1$. The total variance $I$ provides a quantitative measure of the quantum correlations between the quadratures of the distilled states. For symmetric two-mode squeezed Gaussian states, $I$ fully quantifies the entanglement of the state ${ }^{29}$. It has been shown numerically ${ }^{9}$ that, even for the non-Gaussian phase-diffused squeezed states there is a one-to-one correspondence between the reduction of the total variance and the increase of the logarithmic negativity, which is a computable entanglement measure ${ }^{30}$.

Received 15 April 2008; accepted 26 September 2008; published 2 November 2008.

\section{References}

1. Briegel, H.-J., Dür, W., Cirac, J. I. \& Zoller, P. Quantum repeaters: The role of imperfect local operations in quantum communication. Phys. Rev. Lett. 81, 5932-5935 (1998).

2. Duan, L.-M., Lukin, M. D., Cirac, J. I. \& Zoller, P. Long-distance quantum communication with atomic ensembles and linear optics. Nature 414, 413-418 (2001).

3. Julsgaard, B., Sherson, J., Cirac, J. I., Fiurášek, J. \& Polzik, E. S. Experimental demonstration of quantum memory for light. Nature 432, 482-486 (2004).

4. Bennett, C. H. et al. Purification of noisy entanglement and faithful teleportation via noisy channels. Phys. Rev. Lett. 76, 722-725 (1996).

5. Deutsch, D. et al. Quantum privacy amplification and the security of quantum cryptography over noisy channels. Phys. Rev. Lett. 77, 2818-2821 (1996).

6. Zukowski, M., Zeilinger, M. A., Horne, A. \& Ekert, A. K. Event-ready-detectors' Bell experiment via entanglement swapping. Phys. Rev. Lett. 71, 4287-4290 (1993).

7. Browne, D. E., Eisert, J., Scheel, S. \& Plenio, M. B. Driving non-Gaussian to Gaussian states with linear optics. Phys. Rev. A 67, 062320 (2003).

8. Eisert, J., Browne, D. E., Scheel, S. \& Plenio, M. B. Distillation of continuous-variable entanglement with optical means. Ann. Phys. 311, 431-458 (2004).
9. Fiurášek, J., Marek, P., Filip, R. \& Schnabel, R. Experimentally feasible purification of continuous-variable entanglement. Phys. Rev. A 75, 050302(R) (2007).

10. Pan, J.-W., Gasparoni, S., Ursin, R., Weihs, G. \& Zeilinger, A. Experimental entanglement purification of arbitrary unknown states. Nature 423, 417-422 (2003).

11. Zhao, Z., Yang, T., Chen, Y.-A., Zhang, A. N. \& Pan, J.-W. Experimental realization of entanglement concentration and a quantum repeater. Phys. Rev. Lett. 90, 207901 (2003).

12. Furusawa, A. et al. Unconditional quantum teleportation. Science 282, 706-709 (1998)

13. Takei, N., Yonezawa, H., Aoki, T. \& Furusawa, A. High-fidelity teleportation beyond the no-cloning limit and entanglement swapping for continuous variables. Phys. Rev. Lett. 94, 220502 (2005).

14. Jia, X. et al. Experimental demonstration of unconditional entanglement swapping for continuous variables. Phys. Rev. Lett. 93, 250503 (2004).

15. Julsgaard, B., Kozhekin, A. \& Polzik, E. S. Experimental long-lived entanglement of two macroscopic objects. Nature 413, 400-403 (2001).

16. Sherson, J. F. et al. Quantum teleportation between light and matter. Nature 443, 557-560 (2006).

17. Grosshans, F. et al. Quantum key distribution using Gaussian-modulated coherent states. Nature 421, 238-241 (2003).

18. Eisert, J., Scheel, S. \& Plenio, M. B. Distilling Gaussian states with Gaussian operations is impossible Phys. Rev. Lett. 89, 137903 (2002).

19. Giedke, G. \& Cirac, J. I. Characterization of Gaussian operations and distillation of Gaussian states Phys. Rev. A 66, 032316 (2002)

20. Glöckl, O., Andersen, U. L., Filip, R., Bowen, W. P. \& Leuchs, G. Squeezed-state purification with linear optics and feedforward. Phys. Rev. Lett. 97, 053601 (2006).

21. Heersink, J. et al. Distillation of squeezing from non-Gaussian quantum states. Phys. Rev. Lett. 96, 253601 (2006).

22. Franzen, A., Hage, B., DiGuglielmo, J., Fiurášek, J. \& Schnabel, R. Experimental demonstration of continuous variable purification of squeezed states. Phys. Rev. Lett. 97, 150505 (2006)

23. Hage, B. et al. On the distillation and purification of phase-diffused squeezed states. New J. Phys. 9 , 227 (2007).

24. Ourjoumtsev, A., Tualle-Brouri, R., Laurat, J. \& Grangier, P. Generating optical Schrödinger kitten for quantum information processing. Science 312, 83-86 (2006).

25. Neergaard-Nielsen, J. S., Nielsen, B. M., Hettich, C., Mølmer, K. \& Polzik, E. S. Generation of a superposition of odd photon number states for quantum information networks. Phys. Rev. Lett. 97, 083604 (2006).

26. Wakui, K., Takahashi, H., Furusawa, A. \& Sasaki, M. Photon subtracted squeezed states generated with periodically poled $\mathrm{KTiOPO}_{4}$. Opt. Express 15, 3568-3574 (2007).

27. DiGuglielmo, J., Hage, B., Franzen, A., Fiurášek, J. \& Schnabel, R. Experimental characterization of Gaussian quantum-communication channels. Phys. Rev. A 76, 012323 (2007).

28. Duan, L.-M., Giedke, G., Cirac, J. I. \& Zoller, P. Inseparability criterion for continuous variable systems. Phys. Rev. Lett. 84, 2722-2725 (2000).

29. Giedke, G., Wolf, M. M., Krüger, O., Werner, R. F. \& Cirac, J. I. Entanglement of formation for symmetric Gaussian states. Phys. Rev. Lett. 91, 107901 (2003).

30. Vidal, G. \& Werner, R. F. Computable measure of entanglement. Phys. Rev. A 65, 032314 (2002).

\section{Acknowledgements}

J.F. acknowledges the financial support of the Future and Emerging Technologies (FET) programme within the Seventh Framework Programme for Research of the European Commission, under the FET-Open grant agreement COMPAS, number 212008, and from MSMT under projects Center of Modern Optics (LC06007) and Measurement and Information in Optics (MSM6198959213). R.S. acknowledges financial support from the Deutsche Forschungsgemeinschaft (DFG), project No. SCHN 757/2-1.

Author information

Reprints and permissions information is available online at http://npg.nature.com/reprintsandpermissions. Correspondence and requests for materials should be addressed to R.S. 